Review began 02/04/2022 Review ended 02/25/2022 Published 03/03/2022

๑) Copyright 2022

Lin et al. This is an open access article distributed under the terms of the Creative Commons Attribution License CC-BY 4.0., which permits unrestricted use, distribution, and reproduction in any medium, provided the original author and source are credited.

\section{Role of Submandibular Ultrasound in Airway Management of a Patient With Angioedema}

\author{
Dora H. Lin ${ }^{1}$, Brittany Meyers ${ }^{1}$, Saira Nisar ${ }^{1}$, Eric R. Heinz ${ }^{1}$ \\ 1. Anesthesiology and Critical Care Medicine, George Washington University School of Medicine and Health Sciences, \\ Washington DC, USA
}

Corresponding author: Eric R. Heinz, heinz.md.phd@gmail.com

\begin{abstract}
Angioedema is one of several life-threatening clinical scenarios that lacks clarity on when a patient requires intubation. We present a case of angiotensin-converting enzyme-inhibitor-induced angioedema with perioral swelling and normal airway measurements on ultrasound, who was intubated with an abundance of caution and extubated successfully. Current tests for intubation and extubation, such as traditional bedside assessments and the cuff leak test, vary in reliability for angioedema and similar urgent situations.

Submandibular ultrasound is a quick, low-cost, non-invasive method for determining quantitative criteria for and assessing when intubation and extubation is indicated, which may lead to improved quality of care and patient safety.
\end{abstract}

Categories: Anesthesiology, Emergency Medicine, Healthcare Technology

Keywords: extubation failure, difficult extubation, difficult airway management, pocus (point of care ultrasound), angioedema

\section{Introduction}

Angioedema is a life-threatening condition due to blood vessel dilation and increased vascular permeability that causes swelling of the skin and mucosa [1]. While angioedema can be hereditary or acquired [1], it most commonly leads to emergency department (ED) visits when patients are taking an angiotensin-converting enzyme-inhibitor (ACEi) [2]. Around $0.1 \%-0.7 \%$ of those starting an ACEi develop angioedema [3], which is a substantial number of cases given its widespread use. In 2019, over 25 million patients in the United States filled a prescription for an ACEi [4]. ACEi-induced angioedema can lead to swelling of the face, lips, tongue, larynx, pharynx, and neck, as well as difficulty swallowing, speaking, and handling oral secretions $[5,6]$. The majority of cases present within the first few weeks after starting use [1].

Currently, endotracheal intubation is often indicated to protect the airway and occurs in 10\%-34\% of patients presenting to the ED with angioedema [7]. These patients tend to have worsening edema of the oral cavity, pharynx, and larynx and present with drooling, dysphagia, stridor, hoarseness, and dyspnea [8]. However, it may still be unclear at presentation whether a patient requires intubation, and it is often performed out of caution in the event the patient develops respiratory failure. Over the past two decades, there has been a growing interest in the use of point-of-care ultrasound (POCUS) to assess patients perioperatively and in urgent clinical scenarios [9]. Recently, interest has expanded to include using submandibular POCUS to predict difficult airways $[10,11]$. In this case report, we used submandibular ultrasound to scan the oral cavity prior to intubating a patient with ACEi-induced angioedema.

\section{Case Presentation}

A 52-year-old African-American obese man presented to the Emergency Department with angioedema (Figure 1). He has a past medical history of hypertension and abdominal aortic aneurysm, with initiation of lisinopril two weeks prior to presentation. He presented with drooling, dysphagia, and sore throat, but denied tongue swelling, cough, or dyspnea. 


\section{Cureus}

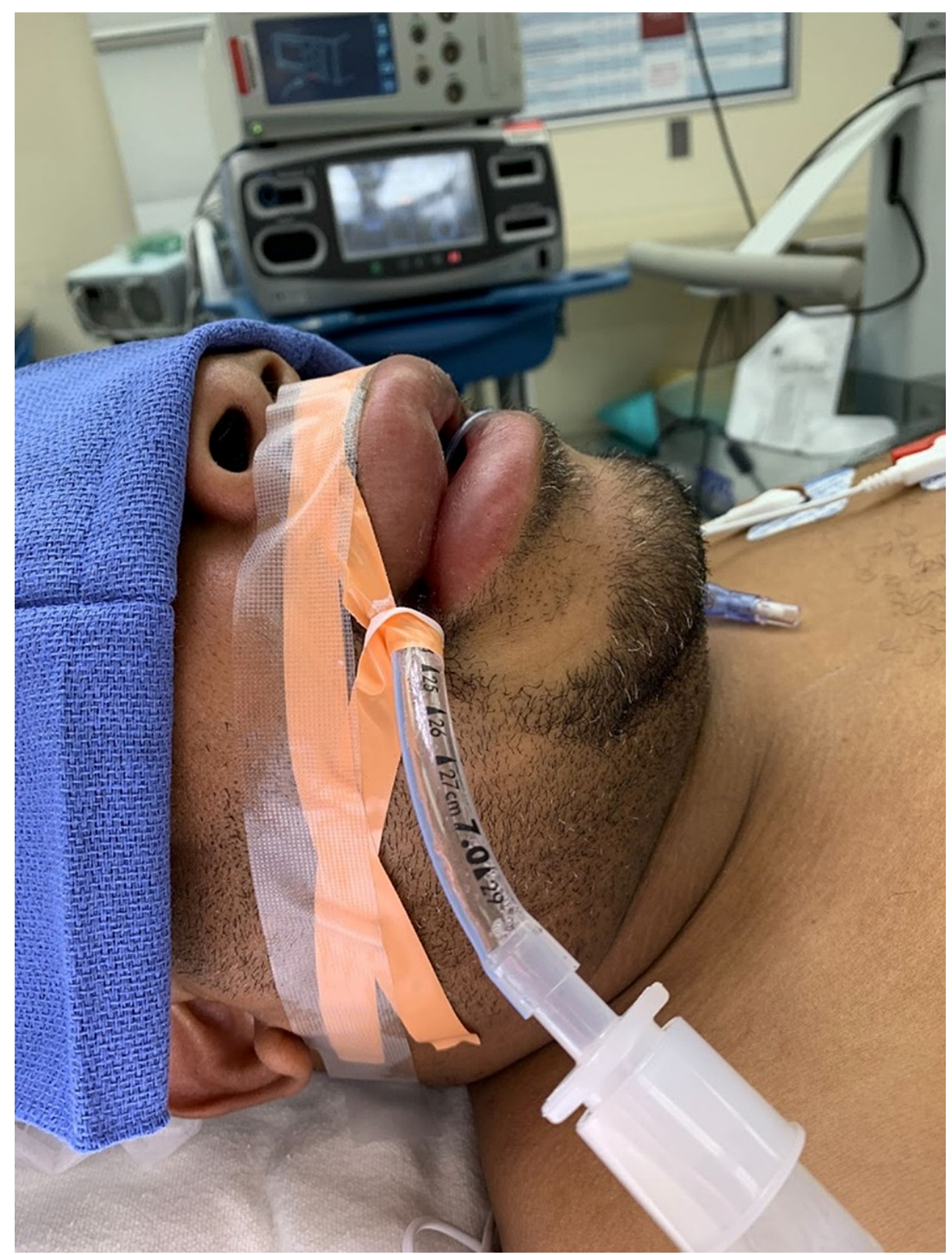

\section{FIGURE 1: Intubated patient with angioedema of the upper and lower}

lips.

On physical examination, he was afebrile, normocardic, and hypertensive, with oxygen saturation $>95 \%$ on room air. He had angioedema of the upper and lower lips, lower face, and eyelid. The angioedema of the lips was primarily right-sided and more upper than the lower lip. Though he had significant angioedema of the lower face, the submental area remained soft. Given that his oral cavity was swollen, traditional bedside airway assessment (including Mallampati score, mouth opening, upper lip bite) was difficult to assess. Therefore, using submandibular ultrasound, we examined the airway and found that the distance between his dorsal lingual arteries was $2.88 \mathrm{~cm}$ and tongue thickness was $4.76 \mathrm{~cm}$ (Figure $2 A-2 D$, Table 1). 


\section{Cureus}
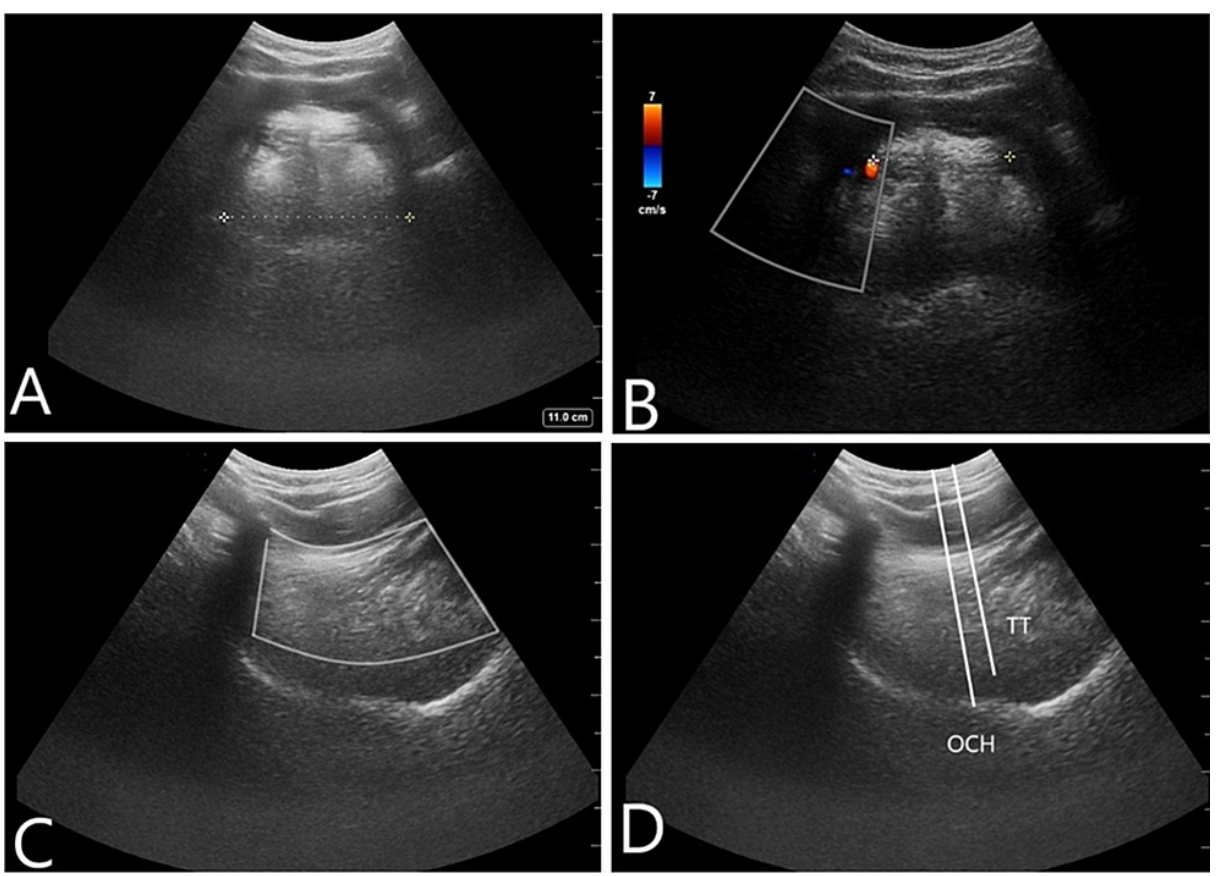

FIGURE 2: Submandibular ultrasound of the patient's airway prior to intubation. (A) Tongue width. (B) Distance between lingual arteries. (C) Tongue cross-sectional area. (D) Tongue thickness and oral cavity height.

TT: tongue thickness; $\mathrm{OCH}$ : oral cavity height.

\begin{tabular}{|l|l|l|}
\hline & Measurement & Normal Mean (SD)* \\
\hline Oral cavity height $(\mathrm{cm})$ & 5.54 & $3.65(0.54)[10]$ \\
\hline Distance between lingual arteries $(\mathrm{cm})$ & 2.88 & $2.89(0.56)[11], 3.04(0.28)[12]$ \\
\hline Tongue thickness $(\mathrm{cm})$ & 4.76 & $3.04(0.48)[10], 4.43(0.63)[13], 6.39(0.51)[11]$ \\
\hline Tongue width $(\mathrm{cm})$ & 5.21 & $4.82(0.50)[12]$ \\
\hline Tongue cross-sectional area $\left(\mathrm{cm}^{2}\right)$ & 19 & \\
\hline Tongue volume $\left(\mathrm{cm}^{3}\right)$ & 98.99 & $0.83(0.03)[10]$ \\
\hline Tongue thickness to oral cavity height ratio & 0.86 & \\
\hline
\end{tabular}

\section{TABLE 1: Patient's upper airway measurements using submandibular ultrasound.}

* Mean and standard deviations previously reported from a few studies, as referenced in this column. Mean (SD) values from Lin et al. (2021) were from those who did not have difficult mask ventilation [11].

He was given intramuscular epinephrine $0.3 \mathrm{mg}$ intravenous (IV), methylprednisolone $125 \mathrm{mg}$ IV, and famotidine 20mg IV. Given the patient's increased drooling, slurred speech, and worsening edema of the upper lip while in the emergency department, consent for intubation was obtained from the patient after explaining the risks and benefits. He was brought to the operating room (OR) for emergent intubation.

Upon arrival in the OR, the patient's airway was scanned using a SonoSite X-porte Ultrasound system (FujiFilm, Philips Healthcare, Bothell, WA) equipped with a 3 to $8 \mathrm{MHz}$ curvilinear transducer. Additionally, he was started on dexmedetomidine infusion for sedation. In preparation for awake fiberoptic intubation, his upper airway was anesthetized with oral 5\% topical lidocaine ointment and nebulized $4 \%$ topical lidocaine solution. A large base of tongue was visualized without significant compression of the epiglottis. 
There was no edema of the oropharynx or larynx. The false and true vocal cords were visualized bilaterally with good vocal cord abduction and no sign of subglottic lesions through the vocal cords. Once the fiberoptic bronchoscope was inserted into the trachea and tracheal rings could be identified, a $7.0 \mathrm{~cm}$ endotracheal tube was successfully placed without complication. The patient was then transferred to the intensive care unit for further management.

His angioedema was treated with IV methylprednisolone $125 \mathrm{mg}$ every 6 hours, oral (PO) famotidine $20 \mathrm{mg}$ twice daily, and IV diphenhydramine $25 \mathrm{mg}$ every 6 hours. He was sedated on IV propofol ( $70 \mathrm{mcg} / \mathrm{kg} / \mathrm{min})$ and IV dexmedetomidine $(0.4 \mathrm{mcg} / \mathrm{kg} / \mathrm{hr})$. Angioedema of the lips and lower face improved by the morning of hospital day 3 . The patient passed the cuff leak test and respiratory rate-tidal volume ratio test and was subsequently successfully extubated to nasal cannula on hospital day 3 . The patient gave written and verbal consent to publish this de-identified case report.

\section{Discussion}

We present the case of a patient with ACEi-induced angioedema who was emergently intubated in the OR. The decision to intubate was made due to his clinical symptoms which include drooling, worsening oral cavity swelling, and dysphagia - all of which have been found in patients with angioedema to be associated with the need to intubate [8].

Generally, it is difficult to assess the necessity to intubate patients with angioedema, given that not all patients with angioedema have airway compromise and require intubation. A few different approaches for deciding whether to intubate include identifying locations of angioedema and presenting symptoms strongly associated with resulting intubation, as well as determining staging criteria $[6,8,13]$. However, the proposed staging criteria for angioedema have been based on visual assessment of the upper airway [13]. According to one staging criteria, our patient would be categorized as stage 2 angioedema due to edema of the soft palate, a stage where none of the patients were intubated in this study [12]. Even for more severe stages, less than a quarter of stage 3 and less than half of stage 4 patients required an airway intervention [13]. As such, it is possible that quantitative measurements, like those offered by submandibular ultrasound [10-12], could help elucidate clearer thresholds for intubation.

In our patient, we found that swelling of the oral cavity precluded proper airway assessment using traditional bedside means such as Mallampati score, mouth opening, and upper lip bite. These bedside clinical tests have poor sensitivities and fair specificities, which have been shown to improve with the addition of ultrasound imaging [14]. Thus, we used submandibular ultrasound to further assess his airway. To our knowledge, POCUS in the ED has been reported only once before as having been used for a patient with angioedema [15]. In this previous case, the authors used anterior neck ultrasound to measure laryngeal edema, while we used submandibular ultrasound to measure tongue and oral cavity swelling [15].

On ultrasound, we found the distance between the patient's lingual arteries (DLA) to be $2.88 \mathrm{~cm}$ and tongue thickness to be $4.76 \mathrm{~cm}$, which are within normal [11,12]. Increasing DLA has previously been reported to be strongly correlated with increasing severity of obstructive sleep apnea, a known cause of difficult intubation [12]. Interestingly, one study of 41 patients reported that tongue base thickness, rather than DLA, is correlated with difficult mask ventilation [11]. These findings suggest that multiple measurements via submandibular ultrasound could be used to determine if intubation is needed and should be further explored. However, we note that the mean tongue thickness was different across previous studies using submandibular ultrasound (Table 1), which may be due to interoperator variability and different methodology used to take measurements [10-12]. Refining the method of submandibular ultrasound measurements can help decrease such variability.

Similarly, it can be difficult to determine when to extubate a patient with angioedema - namely when there is no obstruction of the upper airway [16]. A handful of clinical criteria are currently used to predict the safety of extubation, including the cuff leak test, respiratory rate-tidal volume ratio, and bedside direct laryngoscopy. We decided to extubate our patient after 36 hours, which is close to the average intubation duration in angioedema [16]. He had a negative cuff leak test and sufficiently low respiratory rate-tidal volume ratio (RVR). Moreover, we treated our patient with famotidine and patients with angioedema treated with H1-blockers have been found to be extubated earlier [6]. Our patient was successfully extubated.

However, while a positive cuff leak test may be helpful for identifying airway obstruction, a wide variety of criteria have been used for what is considered positive [17]. Furthermore, findings on whether a negative cuff leak test is reliable for extubation have varied greatly $[18,19]$. With regards to the RVR, it is a reliable test to reflect adequate respiratory muscle strength and endurance to wean from mechanical ventilation [20]. Nonetheless, intubation for ACEi-induced angioedema is related to upper airway edema and obstruction, instead of respiratory spontaneity or muscle strength. Direct laryngoscopy is used for directly visualizing the airway yet is invasive and very noxious to the patient.

We posit that submandibular ultrasound can be a highly beneficial, non-invasive method for deciding not only when to intubate, but also when to extubate the patient with angioedema. As ultrasound is becoming 
more available and utilized in perioperative care, this modality is limited by provider training, experience and comfort level. In retrospect, we could use ultrasound prior to extubating the patient to visually and quantitatively examine airway edema. Given the current uncertainty around airway management in upper airway obstruction, submandibular ultrasound should be further investigated for urgent clinical settings, such as angioedema.

\section{Conclusions}

Submandibular ultrasound is a quick, low-cost, non-invasive imaging modality that may improve quality of care by determining whether a patient needs an airway intervention in unclear clinical presentations, such as angioedema. Our case is an example of a situation that could benefit from the quantitative measurements that submandibular ultrasound can offer, in combination with developing clearer thresholds for intubation and extubation. Future studies should explore the inclusion of submandibular ultrasound measurements as part of the clinical indications that guide urgent clinical scenarios with upper airway obstruction.

\section{Additional Information \\ Disclosures}

Human subjects: Consent was obtained or waived by all participants in this study. Conflicts of interest: In compliance with the ICMJE uniform disclosure form, all authors declare the following: Payment/services info: All authors have declared that no financial support was received from any organization for the submitted work. Financial relationships: All authors have declared that they have no financial relationships at present or within the previous three years with any organizations that might have an interest in the submitted work. Other relationships: All authors have declared that there are no other relationships or activities that could appear to have influenced the submitted work.

\section{References}

1. Lang DM, Aberer W, Bernstein JA, et al.: International consensus on hereditary and acquired angioedema . Ann Allergy Asthma Immunol. 2012, 109:395-402. 10.1016/j.anai.2012.10.008

2. Banerji A, Clark S, Blanda M, LoVecchio F, Snyder B, Camargo CA Jr: Multicenter study of patients with angiotensin-converting enzyme inhibitor-induced angioedema who present to the emergency department. Ann Allergy Asthma Immunol. 2008, 100:327-332. 10.1016/S1081-1206(10)60594-7

3. Byrd JB, Adam A, Brown NJ: Angiotensin-converting enzyme inhibitor-associated angioedema. Immunol Allergy Clin North Am. 2006, 26:725-737. 10.1016/j.iac.2006.08.001

4. ClinCalc DrugStats Database, Version 2021.10. (2021). Accessed: January 14, 2022: https://clincalc.com/DrugStats.

5. Kostis JB, Kim HJ, Rusnak J, Casale T, Kaplan A, Corren J, Levy E: Incidence and characteristics of angioedema associated with enalapril. Arch Intern Med. 2005, 165:1637-1642. 10.1001/archinte.165.14.1637

6. Grant NN, Deeb ZE, Chia SH: Clinical experience with angiotensin-converting enzyme inhibitor-induced angioedema. Otolaryngol Head Neck Surg. 2007, 137:931-935. 10.1016/j.otohns.2007.08.012

7. Smith A, Ray M, Jain N, Zhang H, Sebelik M: The burden of angioedema on United States emergency departments: 2006-2010. Laryngoscope. 2017, 127:828-834. 10.1002/lary.26336

8. Zirkle M, Bhattacharyya N: Predictors of airway intervention in angioedema of the head and neck . Otolaryngol Head Neck Surg. 2000, 123:240-245. 10.1067/mhn.2000.107515

9. Byrne M, Singleton M, Kalagara H, Haskins SC: Perioperative point-of-care ultrasound. Adv Anesth. 2021, 39:189-213. 10.1016/j.aan.2021.07.011

10. Helbing A, Lee E, Pla R, Heinz E: Utilization of submandibular ultrasound to measure oral cavity changes with interventions in routine airway management. Proc (Bayl Univ Med Cent). 2022, 35:20-23. 10.1080/08998280.2021.1980318

11. Lin HY, Tzeng IS, Hsieh YL, Kao MC, Huang YC: Submental ultrasound is effective in predicting difficult mask ventilation but not in difficult laryngoscopy. Ultrasound Med Biol. 2021, 47:2243-2249. 10.1016/j.ultrasmedbio.2021.04.004

12. Bilici S, Engin A, Ozgur Y, Ozlem Onerci C, Ahmet Gorkem Y, Aytul Hande Y: Submental ultrasonographic parameters among patients with obstructive sleep apnea. Otolaryngol Head Neck Surg. 2017, 156:559-566. $10.1177 / 0194599816684109$

13. Dass C, Mahaffa M, Dang E, Campbell R, Ballas Z, Lee S: Evaluation of staging criteria for disposition and airway intervention in emergency department angioedema patients. Acute Med Surg. 2021, 8:e704. 10.1002/ams2.704

14. Andruszkiewicz P, Wojtczak J, Sobczyk D, Stach O, Kowalik I: Effectiveness and validity of sonographic upper airway evaluation to predict difficult laryngoscopy. J Ultrasound Med. 2016, 35:2243-2252. 10.7863/ultra.15.11098

15. Schick M, Grether-Jones K: Point-of-care sonographic findings in acute upper airway edema . West J Emerg Med. 2016, 17:822-826. 10.5811/westjem.2016.9.31528

16. Floyd E, Goldstein NA, Joks R, et al.: An extubation protocol for angioedema. OTO Open. 2017, 1:2473974X17691230. 10.1177/2473974X17691230

17. Ochoa ME, Marín Mdel C, Frutos-Vivar F, Gordo F, Latour-Pérez J, Calvo E, Esteban A: Cuff-leak test for the diagnosis of upper airway obstruction in adults: a systematic review and meta-analysis. Intensive Care Med. 2009, 35:1171. 10.1007/s00134-009-1501-9

18. Shin SH, Heath K, Reed S, Collins J, Weireter LJ, Britt LD: The cuff leak test is not predictive of successful extubation. Am Surg. 2008, 74:1182-1185. 10.1177/000313480807401210

19. Zhou T, Zhang HP, Chen WW, et al.: Cuff-leak test for predicting postextubation airway complications: a 


\section{Cureus}

systematic review. J Evid Based Med. 2011, 4:242-254. 10.1111/j.1756-5391.2011.01160.

20. Chatila W, Jacob B, Guaglionone D, Manthous CA: The unassisted respiratory rate-tidal volume ratio accurately predicts weaning outcome. Am J Med. 1996, 101:61-67. 10.1016/s0002-9343(96)00064-2 